\title{
Editorial
}

\section{Editorial and introduction to Asian Journal of Andrology Prostate Cancer special issue}

\author{
Asian Journal of Andrology (2009) 11: 6-8. doi: 10.1038/aja.2008.40; published online 22 December 2008
}

This is a very special issue of the Asian Journal of Andrology (AJA), which has recently become a part of the Nature Publishing Group's stable. Prostate cancer is unquestionably the most controversial of all the solid malignancies, and we were both proud to be asked to serve as guest editors for this one-off publication. Accordingly, we have commissioned reviews from some of the best researchers and most experienced clinicians and combined these with state-of-the-art research papers. Prostate cancer is best regarded as a continuum, from the earliest microfoci of malignancy to hormone-relapsed disease, and in this special issue we have tried to cover as many aspects as possible of this progressive disease.

This editorial aims to set the scene and introduce a complex subject that is examined in far greater detail in the commissioned reviews and original papers that follow. Worldwide, prostate cancer is the most commonly diagnosed malignancy to afflict men beyond middle age, and is second only to lung cancer as a cause of cancer deaths. Although the incidence continues to rise worldwide, mortality from prostate cancer in the USA recently reached a plateau and has started to decline, but at a rate four times faster than that in the UK [1]. Some have attributed this drop to the efforts made in North America to detect prostate cancer early, although other factors, including more effective treatment, may also have contributed. Although the incidence of prostate cancer is lower in Asian countries than that in the West, the incidence in the East is rapidly rising. Take Shanghai in China as an example; it is very worrying that the average annual incidence of prostate cancer rose from $1.833 / 10^{6}$ in $1984-1989$ to $9.59 / 10^{6}$ in $2005[2,3]$.

Age is the strongest risk factor for prostate cancer, but a family history of the disease affecting one or more firstdegree relatives increases risk by around 2.8 times. Obesity and a Western-style diet high in saturated fats are significant risk factors for the development of prostate cancer. The very latest information on the genetic basis of prostate cancer is reported in the review by Guy et al. [4] in this issue. A large number of studies have evaluated the effects of diet manipulation/supplementation or drug treatment to reduce the incidence of prostate cancer, but the evidence for their efficacy is still weak. The $5 \alpha$-reductase inhibitor finasteride has been shown to reduce the incidence of prostate cancer by $24.8 \%$ compared with placebo over a 7-year period, although at the cost of a small incidence of sexual side effects and a possible increase in the Gleason pattern of the cancers diagnosed in the treated group [5].

In general, the earlier prostate cancer is detected, the better the outlook for the patient in terms of cure or arresting cancer progression. An increasing majority of patients present simply with an isolated increase in prostate-specific antigen (PSA); however, there is no firm evidence that universal screening will reduce mortality, although trials are ongoing. The measurement of PSA, a glycoprotein responsible for liquefying semen, is currently the most effective single screening test for the early detection of prostate cancer. Approximately $25 \%$ of men with PSA levels above the normal range $\left(\geq 4 \mathrm{ng} \mathrm{mL} L^{-1}\right)$ have prostate cancer, and the risk increases to more than $60 \%$ in men with PSA levels above $10 \mathrm{ng} \mathrm{mL}^{-1}$. However, a recent study in prostate cancer prevention, in which all men in the placebo group received a biopsy, reported that the incidence of prostate cancer in men with PSA less than $4 \mathrm{ng} / \mathrm{mL}$ and normal results on digital rectal examination (DRE) was not inconsiderable [5]. A particularly difficult clinical situation occurs when the PSA continues to rise in spite of a previous negative biopsy. A new molecular marker, known as PCA3, may be especially useful in this respect. A urine sample taken immediately after a prostatic massage is analysed. A value of greater than 35 indicates an increased risk that the prostate harbours a malignancy. Furthermore, this new marker may be useful in stratifying men with clinically significant tumours from those with low-risk cancers, which can be managed conservatively [6].

As described here by Borley and Feneley [7], transrectal ultrasonography (TRUS) is the most commonly used modality to image the prostate gland and also to direct the prostate biopsy needle. Antibiotic treatment, usually with a quinolone such as ciprofloxacin, is given before and after the procedure to reduce the risk of infection, which is currently estimated at around 2\%. Usually 8-14 TRUS-guided biopsies are taken from different regions of the prostate with an 18gauge needle. This procedure is now routinely performed on an outpatient basis, preferably after infiltration with local anaesthesia. Histological analysis confirms the presence or absence of cancer and provides a so-called Gleason score for each core. The higher the Gleason score, the greater the risk of disease progression and metastasis.

Magnetic resonance imaging (MRI) is widely used to stage prostate cancer locally and to diagnose or exclude lymph node metastases. Promising results have been reported with the use of ultra-small super-paramagnetic iron oxide particles as an aid to nodal metastasis evaluation by MRI. The addition of magnetic resonance (MR) spectroscopy (evaluation of 
chemical metabolites in a small volume of interest using MR technology) has also improved the accuracy of MR staging.

Radionuclide bone scanning is usually performed as a baseline assessment at the time of the initial diagnosis of prostate cancer. Bone metastases show up as "hot spots" affecting the vertebrae, pelvis or long bones. If the PSA value is $<10 \mathrm{ng} \mathrm{mL}^{-1}$ and the Gleason score is below 8 , it may be permissible to omit this test, as it is rarely positive in these circumstances. The use of this technique in routine follow-up has declined as PSA measurements have been shown to be the most accurate and cost-effective means of monitoring bony metastases.

The aim of treatment in many patients with localised prostate cancer is cure by eliminating the tumour, however, in older patients more conservative methods are often appropriate as many men die with rather than of this disease. Because most men with localised disease do not experience significant disease-related morbidity for several years after diagnosis, and curative treatment itself may result in some morbidity, those with a shorter life expectancy are likely to benefit least from radical treatment. Radical prostatectomy involves surgically removing the entire prostate, the seminal vesicles and a variable amount of adjacent tissue. It is most appropriate for men for whom it is believed the tumour can be removed completely by surgery and who have no co-morbidity. The procedure is most commonly performed via the retropubic route, although the perineal approach can also be used. The major advantage of radical prostatectomy is that it excises all prostatic tissue and provides precise histological information and definitive cure in patients in whom the tumour is specimen-confined. Thus, the patient's anxiety is relieved during the postoperative period; given that prostate cancer has a long natural history, this is an important consideration in terms of quality of life. Long-term studies have shown normal life expectancies in those with complete excision of specimen-confined disease. Ten-year survival for men with clinically localised disease treated with radical prostatectomy is $98 \%, 91 \%$ and $76 \%$ for Gleason scores of $2-4,5-7$ and $8-10$, respectively. Moreover, the procedure also offers definitive treatment of concomitant benign prostate hyperplasia.

The principal adverse events associated with radical prostatectomy are persistent stress urinary incontinence $(<$ $2 \%-3 \%)$ and erectile dysfunction ( $>50 \%$ ); the latter is age-related, tends to improve with time and can be minimised by nerve-sparing approaches. Moreover, erectile dysfunction after surgery can now be treated effectively. Radical prostatectomy, by whichever means achieved, is believed by many urologists to offer the best opportunity for cure in patients with localised prostate cancer. A randomised study in Sweden showed that a median 8.2 years' followup, radical prostatectomy decreased prostate-cancer-related mortality by $44 \%$ and overall death by $26 \%$ [8]. Recently, laparoscopic radical prostatectomy was described. The minimally invasive approach decreases blood loss and postoperative pain. It can be facilitated by robotic assistance with the da Vinci device, which provides 3D vision and 10 times magnification [9]. This special issue contains two specially commissioned articles that review this procedure in detail.

External-beam radiotherapy is widely used in the treatment of localised prostate cancer. It offers a particular advantage in patients who are unsuitable for surgery because of comorbidity or evidence of extraprostatic extension of cancer. As Dr Heather Payne [10] describes in this issue, the treatment generally involves a 7-week course of radiotherapy. Ten-year survival of patients undergoing external-beam radiation for clinically localised prostate cancer who have Gleason scores of $2-4,5-7$ and $8-10$ is reported to be approximately $89 \%, 74 \%$ and $52 \%$, respectively. The principal side effects are due to bladder, urethral and rectal damage from the radiation scatter. Increased urinary frequency and urgency are common. In its severe form, urinary bleeding and pain may occur in $2 \%-3 \%$ of patients. Rectal side effects consist of urgency, frequency and tenesmus. If severe, rectal bleeding, pain or fistula may very occasionally require a colostomy. Erectile dysfunction due to damage to the neurovascular supply to the corpora cavernosa can also occur, typically over a 6-18-month period.

Low-dose seed brachytherapy involves placing either iodine- 125 or palladium-103 seeds into the prostate via the transperineal route using a template and TRUS guidance. The results of seed brachytherapy in low-risk men (PSA $<10 \mathrm{ng} \mathrm{mL}^{-1}$, Gleason score $<7$ and $\leq$ cT2b) are equivalent to those with radical prostatectomy at 10 years, but they are highly dependent on the quality of seed placement. The results in patients with intermediate risk are worse, but approximately $66 \%$ have not had a recurrence at 10 years. The method is popular, particularly in the USA, because of its low morbidity. The side effects include lower urinary tract symptoms that are a direct result of the seeds. Rectal symptoms and incontinence are unusual, but erectile dysfunction may develop over time.

Active surveillance is usually reserved for men with small-volume and low-to-moderate-grade prostate cancer, who have a low risk of death from prostate cancer. Such men would be eligible for curative therapy, but this option is deferred until objective signs of biological activity are observed. This approach means that the majority of men are spared the side effects of curative therapy when they do not require it. During active surveillance, men are followed closely with regular PSA measurements and DREs and repeat prostate biopsies. If the cancer appears to progress significantly, curative therapy is initiated before the cancer becomes incurable [11]. The cancer-specific survival in men who fit the criteria for active surveillance is $99 \%$ at 8 -year follow-up [12]. Although these men avoid the physical side effects of cancer treatment, they must live with the psychological effects of having an untreated cancer.

Although there is a worldwide trend towards earlier detection of prostate cancer, many patients still present with 
metastatic disease. In contrast to localised or locally advanced disease, metastatic prostate cancer is associated with high mortality - approximately 70\% within 5 years. Androgen deprivation can be achieved by orchidectomy or by treatment with a luteinizing hormone-releasing hormone (LHRH) analogue or an antiandrogen. In most cases, advanced prostate cancers treated with any form of androgen deprivation eventually begin to progress, a phenomenon known as 'hormonerefractory' or 'androgen-independent' disease. Docetaxel, a chemotherapeutic agent, was recently tested in a randomised trial against mitoxantrone and prednisone in men with androgen-independent prostate cancer. The results of this study (TAX-327) showed docetaxel, given 3-weekly for up to 10 cycles, to be superior to mitoxantrone/prednisone in decreasing disease progression and PSA response and reducing pain [13]. In addition, docetaxel significantly improved survival from a median of 16.4 months for mitoxantrone/prednisone to 18.9 months for 3-weekly docetaxel, which correlates to a $24 \%$ relative reduction in death. The side effects associated with docetaxel include neutropenia, skin reactions and gastrointestinal problems.

As our readers will be aware, many aspects of prostate cancer diagnosis and management are still highly controversial compared with those of, say, breast cancer [14]. This largely reflects the considerably greater research effort expended on the latter disease and, in particular, the much greater number of randomised controlled trials, which have defined optimal treatment strategies for women with breast cancer. This imbalance is beginning to be redressed, and the situation in prostate cancer is improving. Recent advances, such as the introduction of PCA3 as a new marker, the development of robot-assisted surgery and more effective treatment of hormone-relapsed prostate cancer, are beginning to offer real benefits to patients. Further improvements seem likely as the evidence base improves and new treatment approaches, such as endothelin A antagonists, become available.

Finally, we thank all our authors, all our readers, and all of those-especially the staff of the editorial office of the Asian Journal of Andrology-who worked so hard to turn the concept of this special issue into reality.

\section{Guest Editors}

Prof. Roger Kirby, The Prostate Centre, London, UK (E-mail: rogerkirby@theprostatecentre.com)

Prof. Shu-Jie Xia, Department of Urology, Shanghai First People's Hospital, Shanghai Jiao Tong University, Shanghai, China (E-mail: xsjurologist@163.com )

\section{Acknowledgment}

We would like to make grateful acknowledgement to Gen-Probe for kindly providing the cover image for this special issue.

\section{References}

1. Collin SM, Martin RM, Metcalfe C, Gunnell D, Albertsen PC, et al. Prostate-cancer mortality in the USA and UK in 1975-2004: an ecological study. Lancet Oncol 2008; 9: 445-52.

2. Xu ZB, Wang GM, Sun LA, Zhang YK. The incidence of prostatic carcinoma in Shanghai. Clin Med J Chin 2003; 10: 345-6.

3. Shanghai Municipal Center for Disease Control and Prevention. Incidence of malignant tumors of city Shanghai in the year 2005. Tumor 2007; 27: 634 .

4. Guy M, Kote-Jarai Z, Giles GG, Al Olama AA, Jugurnauth SK, et al. Identification of new genetic risk factors for prostate cancer. Asian J Androl 2009; 11: 49-55.

5. Thompson IM, Goodman PJ, Tangen CM, Lucia MS, Miller GJ, et al. The influence of finasteride on the development of prostate cancer. N Engl J Med 2003; 349: 215-24.

6. Nakanishi H, Groskopf J, Fritsche HA, Bhadkamkar V, Blase A, et al. PCA3 molecular urine assay correlates with prostate cancer tumor volume: implication in selecting candidates for active surveillance. J Urol 2008; 179: 1804-10.

7. Borley N, Feneley MR. Prostate cancer: diagnosis and staging. Asian J Androl 2009; 11: 57-63.

8. Bill-Axelson A, Holmberg L, Filén F, Ruutu M, Garmo H, et al; Scandinavian Prostate Cancer Group Study Number 4. Radical prostatectomy versus watchful waiting in early prostate cancer. N Engl J Med 2005; 352: 1977-84.

9. Goldstraw MA, Patil K, Anderson C, Dasgupta P, Kirby RS. A selected review and personal experience with robotic prostatectomy: implications for adoption of this new technology in the United Kingdom. Prostate Cancer Prostatic Dis 2007; 10: $242-9$.

10. Payne H. Management of locally advanced prostate cancer. Asian J Androl 2009; 11: 64-70.

11. Klotz, L. Active surveillance with selective delayed intervention is the way to manage 'good-risk' prostate cancer. Nat Clin Pract Urol 2005; 2 : $136-42$.

12. Albertsen PC, Hanley JA, Fine J. 20-year outcomes following conservative management of clinically localized prostate cancer. JAMA 2005; 293: 2095-101.

13. Petrylak DP, Tangen CM, Hussain MH, Lara PN Jr, Jones JA, et al. Docetaxel and estramustine compared with mitoxantrone and prednisone for advanced refractory prostate cancer. N Engl J Med 2004; 351: 1513-20.

14. Kirby RS, Partin A, Feneley M, Parsons K. Prostate Cancer: Principles and Practice. Informa plc, London, 2007. 